\title{
IN THIS ISSUE - FEBRUARY 1, 2008
}

\section{JAVMA News}

Helping veterinarians address obesity in cats and dogs is the goal of the new Alliance for Healthier Pets-Obesity Awareness and Prevention Program from the AVMA and Hill's Pet Nutrition.

See PAGE 340

Letters to the Editor

See PAGE 350

\section{What Is Your Diagnosis?}

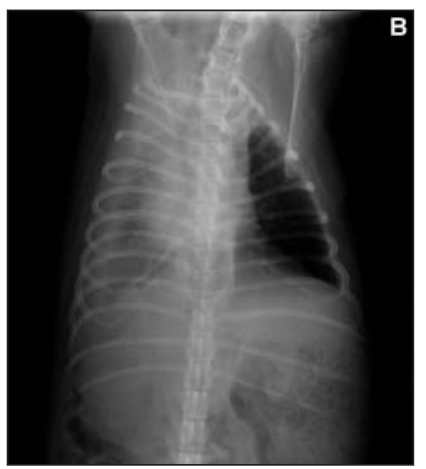

See PAgES 355,357

DISASTER MEDICINE

\section{Decontamination of animals involved in floodwater disasters}

Decontamination of animals exposed to the hazards dispersed by natural disasters is an important component of responsible emergency management and will help to minimize the risk of contaminants spreading through animal intake facilities. Implementing a simple and efficient decontamination protocol will ensure continuous operation even during times of high intake or depleted resources.

See PAGE 364
Prevalences of various hemoplasma species among cats in the United States with possible hemoplasmosis

Information on the prevalence of infection with various hemoplasma species among cats in the United States is lacking. Use of conventional and species-specific real-time PCR assays to analyze blood samples from 310 cats with evidence of hemoplasmosis revealed that $72(23.2 \%)$ were infected with "Candidatus Mycoplasma haemominutum," 15 (4.8\%) were infected with Mycoplasma haemofelis, and $20(6.5 \%)$ were infected with "Candidatus Mycoplasma turicensis." Cats infected with hemoplasmas were more likely to be male than were uninfected cats. Results supported the suggestion that these 3 hemoplasma species commonly occur among cats in the United States and that pathogenicity of the 3 species varies.

See PAGE 372

\section{Surgically placed gastrojejunostomy feeding tubes in critically ill dogs}

In human patients, use of surgically placed gastrojejunostomy tubes to provide supplemental enteral nutrition has been shown to be associated with fewer complications than use of surgically placed jejunostomy tubes. Techniques for insertion of gastrojejunostomy tubes in dogs have been described, but little information is available on outcome of their use. Evaluation of 26 critically ill dogs in which a gastrojejunostomy tube was inserted because of a perceived need for intensive postgas-

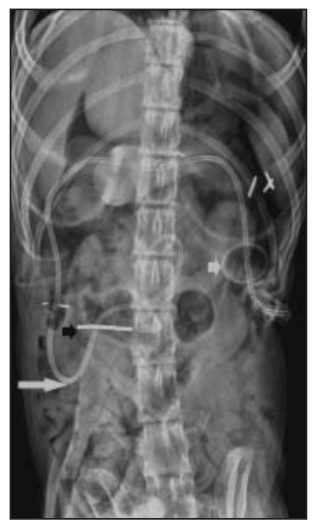
tric nutritional support following surgical treatment of gastrointestinal tract, pancreatic, or extrahepatic biliary disease revealed that this was an effective method of providing postoperative enteral nutritional supplementation with a low rate of serious postoperative complications.

See PAGE 380

\section{Doxycycline in drinking water for treatment of spiral bacterial infection in cockatiels}

Spiral bacterial infections in cockatiels have been successfully treated with oral administration of doxycycline. However, this treatment regimen requires twicedaily capture for a prolonged period for the medication to

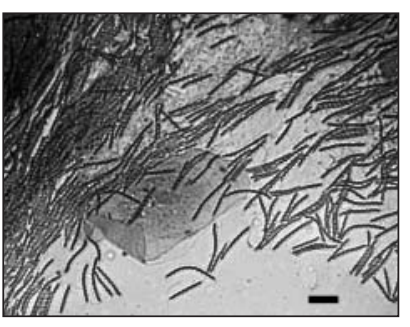
be administered, which can be difficult for some bird owners. To determine whether administration of doxycycline in the drinking water would also be effective, a randomized con- 


\section{February 1, 2008-InTHIS Issue}

trolled trial involving 18 cockatiels was performed. Cockatiels $(n=11)$ given drinking water to which doxycycline had been added for 30 days were negative for spiral bacteria after treatment for 21 days and remained negative when examined 160 days after treatment ended. Control birds (7) remained positive for spiral bacteria.

See PAgE 389

\section{Pituitary-dependent hyperadrenocorticism in a cockatoo}

A 13-year-old Moluccan cockatoo examined because of a presumptive abdominal hernia was also reported to have had a history of rapid weight gain, polyuria, and polydipsia. Clinical signs and results of clinicopathologic evaluations were compatible with a diagnosis of hyperadrenocorticism, although results of ACTH stimulation testing were equivocal. The bird was euthanized because of complications associated with herniorrhaphy, and a diagnosis of pituitary-dependent hyperadrenocorticism was confirmed on the basis of results of histologic examination of the pituitary gland. Findings indicated that pituitary-dependent hyperadrenocorticism can develop in Psittaciformes, causing signs similar to those seen in mammals, and that ACTH stimulation testing in affected birds may yield equivocal results.

See PAGE 394

\section{Marsupialization of a hemorrhagic intramedullary vascular anomaly in a dog}

Magnetic resonance imaging of a 1-year-old dog examined because of progressive tetraparesis and generalized proprioceptive ataxia revealed an intramedullary fluid-filled cavitary lesion adjacent to $\mathrm{C} 7$ that contained a blood clot. Dorsal laminectomy, durotomy, and myelotomy were performed to allow marsupialization of the lesion, and immunohistochemical evaluation of the lesion revealed that it was a vascular anomaly. The dog was able to walk without assistance by 4 weeks after surgery and was actively running and jumping with only mild residual ataxia 1 year after surgery. In this dog, the combination of magnetic resonance imaging and immunohistochemical evaluation was crucial to lesion location and identification.

See PAGE 399

\section{Outcome of cats with low-grade lymphocytic Iymphoma}

Lymphomas in cats can be classified histologically as high, intermediate, or low grade, with only approximately $10 \%$ of lymphomas in cats considered low grade. Many studies have been published

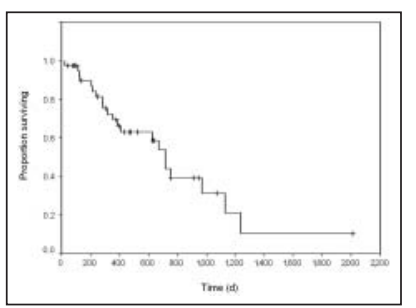

describing the diagnosis, treatment, and prognosis of cats with Iymphoma. However, little information is available specifically on cats with low-grade lymphoma. A review of medical records of 41 cats with low-grade lymphoma revealed that $95 \%$ had a complete or partial response to treatment with prednisone and chlorambucil, with a median overall survival time of 704 days. Median remission duration was 897 days for cats with a complete response to chemotherapy and 428 days for cats with a partial response.

See PAGE 405

\section{Adverse effects of dexamethasone administration in dogs with acute thoracolumbar disk herniation}

Despite numerous reports of the benefits of high-dose glucocorticoid treatment in patients with acute spinal cord injury, substantial data exist to suggest that these benefits may be limited, transient, or nonexistent in the clinical setting. Review of medical records of 161 dogs with thoracolumbar disk herniation revealed that dogs that received dexamethasone $<48$ hours prior to admission were 3.4 times as likely to have complications as were dogs that received some other glucocorticoid or did not receive glucocorticoids. No differences in neurologic function at discharge or recheck evaluation were detected among groups, suggesting that the value of preoperative dexamethasone administration in dogs with thoracolumbar disk herniation should be reconsidered.

See PAGE 411

\section{Effects of erythromycin and flunixin meglumine on postoperative abomasal emptying rate in dairy cows with LDA}

In cows, abomasal emptying rate is decreased immediately after surgical correction of left displacement of the abomasum. Improving abomasal motility after surgery, therefore, could increase dry matter intake, rumen motil-

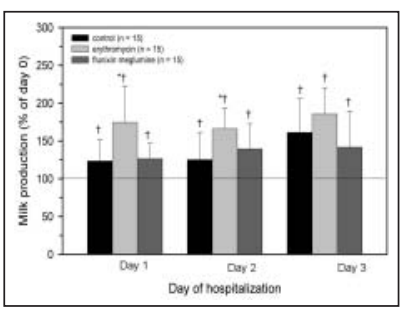
ity, and milk production. In a clinical trial involving 45 dairy cows undergoing surgery for left displacement of the abomasum that were given a single dose of erythromycin or flunixin meglumine prior to surgery to prevent postoperative hypomotility or were not given any specific treatment, preoperative administration of a single dose of erythromycin was found to increase abomasal emptying rate, rumen contraction rate, and milk production in the immediate postoperative period. Administration of flunixin did not increase abomasal emptying rate.

See PAGE 418 